\title{
Effect of 3 months vitamin $E$ supplementation on indices of the cellular and humoral immune response in elderly subjects
}

\author{
BY FROUWKJE G. DE WAART, LÜTZEN PORTENGEN, GERT DOEKES, \\ CORNÉ J. VERWAAL AND FRANS J. KOK \\ Department of Epidemiology and Public Health, Wageningen Agricultural University, \\ PO Box 238, 6700 AE Wageningen, The Netherlands
}

(Received 17 January 1997 - Revised 14 April 1997 - Accepted 1 May 1997)

\begin{abstract}
It has been suggested that decreased immune responsiveness in the elderly may be counteracted by the antioxidant vitamin $E$. In a 3-month double-blind placebo-controlled intervention trial among elderly subjects aged 65 years and over we studied the effects of a daily dose of $100 \mathrm{mg} \mathrm{dl}-\alpha-$ tocopheryl acetate on the cellular immune responsiveness $(n$ 52) measured by the in vitro response of peripheral blood mononuclear cells (PBMC) to the mitogens concanavalin A (ConA) and phytohaemagglutinin (PHA). Also effects on the humoral immune responsiveness $(n$ 74) were investigated by measuring immunoglobulin (Ig)G, IgG4 and IgA antibody concentrations against various common antigens. In the vitamin $E$ group plasma $\alpha$-tocopherol increased by $51 \%$ $(\boldsymbol{P}=\mathbf{0 . 0 0 0 1 )}$ during intervention whereas no significant changes were observed in the control group. Initial proliferative PBMC responses differed between the vitamin $E$ group and the control group whereas all other baseline characteristics were comparable. No significant changes were observed in cellular immune responsiveness when adjusted for initial values in either the control group or the vitamin $E$ group and, after the trial period, responses in the two groups were not significantly different. Similarly, in the vitamin $\mathbf{E}$ group no significant changes were found in levels of IgG and IgA raised against Penicillium or IgG4 raised against egg, milk, or wheat proteins. In the control group small but significant increases in IgG anti-Penicillium $(P<0.05)$ and decreases in IgG4 against milk proteins $(P<0.05)$ were observed. Thus, the results of this study performed with the relatively low dose of $100 \mathrm{mg}$ dl- $\alpha$-tocopheryl acetate do not support the claims of a beneficial effect of vitamin $E$ intake on the overall immune responsiveness of elderly subjects.
\end{abstract}

Vitamin E: Cellular immune response: Humoral immune response: Elderly

A decreased functioning of specific immune defence mechanisms with ageing (Weksler \& Hutteroth, 1974; Kishimoto et al. 1980; Burns et al. 1990; Wayne et al. 1990; Makinodan, 1995 ) is presumed to contribute to increased morbidity and mortality in the elderly (Roberts-Thomson et al. 1974; Gardner, 1980; Murasko et al. 1988; Wayne et al. 1990; Goodwin, 1995). In addition to chronic diseases that accompany ageing that may lead to a decrease in immune responsiveness in the elderly, the ageing process itself is thought to play a major role too (Goodwin et al. 1982), highlighting the potential relevance of improving the immune response in 'healthy' elderly people. Increased lipid peroxidation and production of eicosanoids such as prostaglandin $\mathrm{E}_{2}\left(\mathrm{PGE}_{2}\right)$ may be important determinants in age-associated dysregulation of immune responsiveness. Vitamin $\mathrm{E}$ may improve immune responsiveness by counteracting these effects (Corwin \& Shloss, 1980a; Goetzel, 1980; Likoff et al. 1981; Meydani et al. 1986, 1990, 1995; Hayek et al. 1994). 
Effects of vitamin E supplementation on the cellular and humoral immune response in experimental animals have been reported extensively (Tengerdy et al. 1973, 1984; Corwin \& Shloss, 1980b; Corwin et al. 1981; Larsen \& Tollersrud, 1981; Bendich et al. 1986; Meydani et al. 1986; Reddy et al. 1987). Observational studies in healthy elderly human subjects found no positive associations between vitamin $E$ intake or status and indicators of cell-mediated immune response (Goodwin \& Garry, 1983; Chavance et al. 1989; Payette et al. 1990). However, these observational studies were cross-sectional and not designed to provide information on the effect of (long term) specific vitamin supplementation. In an intervention study among free-living healthy human elderly subjects with a daily dose of $800 \mathrm{mg}$ dl- $\alpha$-tocopheryl acetate for $30 \mathrm{~d}$, Meydani et al. (1990) observed an overall improvement in cell-mediated immune response as shown by increased frequency and size of positive delayed type hypersensitivity skintest responses, interleukin-2 production and in vitro proliferative response of peripheral blood mononuclear cells (PBMC) to the mitogen concanavalin $\mathrm{A}(\mathrm{ConA})$. Also a reduced $\mathrm{PGE}_{2}$ synthesis by $\mathrm{PBMC}$ was seen, which argues in favour of reduction of $\mathrm{PGE}_{2}$ production as a possible working mechanism. In the same study no significant differences were found in humoral immune response measured as total serum concentrations of immunoglobulin (Ig)M, IgG and $\operatorname{IgA}$, nor in the T-cell independent proliferative response of B cells to the mitogen Staphylococcus aureus Cowan. In another intervention study among institutionalized healthy elderly women, supplementation with $200 \mathrm{mg}$ vitamin E for 1 year had no effect on total IgM, IgG and IgA antibody concentrations (Ziemlanski et al. 1986).

The optimal dose for potential beneficial effects of supplemental vitamin $E$ on immune variables in the elderly has not yet been determined. Studies investigating low or relatively (compared with other trials) low doses of supplemental vitamin $\mathrm{E}$ can contribute to the debate on whether it is warranted to advise elderly people to increase their intake of vitamin E-rich foods, or to explore the possibilities of enrichment of eligible food products or to advise vitamin E supplement use. But, at this point there is only limited evidence that the positive effects of vitamin $\mathrm{E}$ on immune responses, as found in animal experiments, generalize to human elderly subjects. Therefore, we have investigated the effects of a 3month period of $100 \mathrm{mg} \alpha$-tocopheryl acetate supplementation on cell-mediated immune responsiveness, expressed as in vitro response of PBMC to the mitogens Con $\mathrm{A}$ and phytohaemagglutinin (PHA), and on humoral immune responsiveness, assessed by measuring IgG, IgG4 and IgA antibody concentrations against various common antigens. To avoid inter-assay variation, inherent to the mitogen proliferation assays, isolated PBMC were stored in liquid $\mathrm{N}_{2}$ and mitogen proliferative response of pre- and post-intervention PBMC of each individual were measured simultaneously in the same run.

\section{METHODS}

\section{Population and design}

The study was designed as a 3-month randomized double-blind placebo-controlled trial to investigate the association between vitamin $E$ and health characteristics in elderly subjects, such as immune response, lung function and, as the main question, the effect on oxidation of LDL-cholesterol, and was conducted in 1994. In two consecutive steps a total of eightythree apparently healthy elderly subjects, aged 67-85 years, were selected from a database of a population of 1012 elderly people in the city of Arnhem, The Netherlands (Van den Homberg, 1995). The first selection step included the following criteria: still living in Arnhem, not institutionalized, no use of prescribed drugs for cardiovascular disease (CVD), high blood pressure or diabetes mellitus; no reported cancer or chronic diseases of 
the bowel, or stomach, kidney, or liver. In the second step, the 355 remaining persons were invited by mail and received a questionnaire on health status, smoking habits, and use of supplements and medication. Seventy-five subjects did not respond, 129 refused and 151 were willing to participate. From this latter group sixty-eight were not eligible because of the following criteria: use of vitamin supplements $(\mathrm{A}, \mathrm{A}+\mathrm{D}, \mathrm{C}, \mathrm{E}$ or multivitamin; $(n 25)$, stopped smoking less than 5 years before $(n 5)$, current treatment for CVD, heart attack or stroke $(n 31)$, use of medication for hypertension or high cholesterol $(n 6)$, or diabetes $(n 2)$. The eighty-three eligible subjects were randomly assigned to intervention ( $n$ 42) and placebo groups ( $n 41$ ). To ensure group comparability, subjects were prestratified before randomization on the following variables: smoking habits (current $v$. non-smokers), 5-year age groups and sex. The subjects received either vitamin $\mathrm{E}(100 \mathrm{mg} \mathrm{dl}-\alpha$-tocopheryl acetate; Hoffman-La Roche, Mijdrecht, The Netherlands; $50 \mathrm{mg}$ twice daily) or placebo (lactose) capsules. Of the eighty-three subjects entering the study one subject in the vitamin E group who took only $15 \%$ of the distributed capsules was excluded from the data analysis. Eight subjects had missing values on baseline or post-intervention humoral immune response measurements. A random group of thirty subjects had missing values for baseline and/or post-intervention values for the mitogenic proliferation responses due to shortage of plasma or isolated blood mononuclear cells to perform all the tests. Data analysis was performed on complete data sets. Therefore, baseline and humoral immune characteristics are presented for seventy-four subjects (thirty-six in the placebo group and thirty-eight in the vitamin E group). For cell-mediated immune response, data on fifty-two subjects (twenty-five placebo and twenty-seven vitamin E group) are presented.

\section{Data collection}

At the beginning and end of the 3-month intervention period subjects were picked up at home and brought to the examination room at the University. Fasting blood samples were taken into EDTA for determination of antioxidant vitamin levels and for measurement of indices of the cellular and humoral immune response. Body weight, height and blood pressure were determined on both occasions as well. At baseline the subjects filled in a questionnaire on socio-demographic characteristics, physical activity and perceived health score. A semi-quantitative food-frequency questionnaire was administered and used to estimate antioxidant vitamin intake (Ocké et al. 1997). Subjects were asked to monitor intercurrent illness and/or medicine use in a diary. To improve and monitor compliance subjects were visited at home once during the trial. At the end of the study compliance was assessed by pill counts.

The study was approved by the Medical Ethical Committee of the Agricultural University and all subjects gave written informed consent to participate.

\section{Laboratory measurements}

Plasma was prepared under $\mathrm{N}_{2}$ within $3 \mathrm{~h}$ after venepuncture and stored at $-80^{\circ}$ until analysis, to minimize analytical variation. Laboratory baseline and post-intervention measurements for each individual were performed in the same run. Concentrations of $\alpha$ tocopherol, $\beta+\gamma$-tocopherol, and $\beta$-carotene were measured in plasma by reverse-phase HPLC (adapted from Hess et al. 1991). The HPLC analyses were performed using a system from Thermo Separation Products (Freemont, CA, USA). The column was a prepacked $0.25 \mathrm{~m} \times 4.6 \mathrm{~mm}$ Vydac 201TP54, $\mathrm{C}_{18} 300 \AA$ (Hesperia, CA, USA). The mobile phase used for the Vydac column was methanol-tetrahydrofuran-water in the following proportions: 
$0 \min , 89: 2: 9 ; 10 \mathrm{~min}, 98: 2: 0 ; 20 \min 97: 3: 0 ; 30 \min 90: 10: 0 ; 40 \mathrm{~min}, 90: 10: 0$ by volume, and the flow rate was $1 \mathrm{ml} / \mathrm{min}$. Detection after separation was carried out using two u.v. detectors, one for determination of carotenoids (u.v. 2000) and one (u.v.1000) for determination of the tocopherols.

Humoral immune response: antigen-specific immunoglobulins $G$ and $A$. The following specific antibodies were measured by enzyme immunoassay essentially as described previously (Houba et al. 1996; Zock et al. 1996): IgG and IgA against a mixture of extracts of four different strains of Penicillium (Allergologisk Laboratorium Kopenhagen, Benelux, Houten, The Netherlands; art. no. 25-00) and IgG4 against egg (Laboratorium Diephuis, Groningen, The Netherlands; art. no. 58-04), milk (art. no. 58-01) and wheat (Houba et al. 1996) proteins. High-capacity microtitre plates (Greiner no. 655061; Greiner, Nuertingen, Germany) were coated overnight at $4^{\circ}$ with the antigen preparations in phosphate-buffered saline (PBS). After blocking of free binding sites with gelatin-containing PBS-Tween (PBTG), duplicate $0.1 \mathrm{ml}$ portions of sera diluted 1:100 (IgG4) and 1:500 (IgA, IgG) in PBTG were incubated in wells coated with the various antigens and in non-coated control wells. Binding of IgG, IgG4 or IgA was measured with peroxidase-labelled mouse monoclonal anti-human IgG (Central Laboratory of the Red Cross Blood transfusion Service, Amsterdam, The Netherlands (CLB) no. M1304), antihuman IgG4 (CLB no. M1331), or anti-IgA (CLB, no. M1354) respectively, followed by 30 min incubation with $o$-phenylenediamine (OPD). The reaction was read at $405 \mathrm{~nm}$, terminated by addition of $\mathrm{HCl}$, and then read at $492 \mathrm{~nm}$. Absorbance values at $492 \mathrm{~nm}$ $\left(A_{492}\right)>3.0$ were obtained by extrapolation from the $A_{405}$ values according to the method of Doekes et al. (1993).

Each plate included a positive control (pooled plasma) and no-plasma controls in each type of coated and non-coated well. Antibody concentrations were expressed as $\mathrm{A}_{492}{ }^{*}$ values corrected for values observed in control wells, as follows:

$$
A_{492} *\left(i, a_{1}\right)=\left[A_{492}\left(i, a_{1}\right)-A_{492}\left(0, a_{1}\right)\right]-\left[A_{492}(i, 0)-A_{492}(0,0)\right]
$$

in which $A_{492}\left(i, a_{1}\right)$ is the value obtained with plasma $i$ on antigen $a_{1}, A_{492}\left(0, a_{1}\right)$ the value obtained with buffer on antigen $a_{1}$, and $A_{492}(i, 0)$ and $A_{492}(0,0)$ are the analogous values obtained in non-coated wells.

Pre- and post-intervention plasma samples of each subject were tested in the same microtitre plate, to avoid inter-assay variation.

Cellular immune response: isolation and mitogenic stimulation of peripheral blood mononuclear cells. Within $6 \mathrm{~h}$ after venepuncture, PBMC were isolated from EDTAblood by Ficoll-Hypaque (Pharmacia BiotechAB, Uppsala, Sweden) density gradient centrifugation (Boyum, 1968). PBMC were harvested and washed twice with sterile PBS and resuspended in $1 \mathrm{ml}$ culture medium (RPMI 1640; Sigma-Aldrich Co. Ltd, Irvine, Strathclyde) with $100 \mathrm{ml} / 1$ dimethylsulfoxide (DMSO) (hybri-max, Sigma-Aldrich Co. Ltd) in polypropylene vials. The cells were frozen overnight at $-80^{\circ}$ and stored in liquid $\mathrm{N}_{2}$ $\left(-196^{\circ}\right)$ until analysis.

For mitogenic stimulation, vials containing pre- and post-intervention PBMC were removed from the liquid $\mathrm{N}_{2}$ simultaneously and thawed quickly at $37^{\circ}$ in a $5 \% \mathrm{CO}_{2}$ incubator. DMSO was removed by washing with RPMI-1640. This washing procedure was repeated twice to ensure all DMSO had been removed, after which the pellets were resuspended in $750 \mu \mathrm{l}$ RPMI-1640 and the concentration of viable cells was determined with the Trypan Blue exclusion method.

Portions of $0.5 \times 10^{6}$ cells were cultured in twenty-four-well plates (Costar, Cambridge, MA, USA) in $0.50 \mathrm{ml}$ RPMI-1640 containing $2 \mathrm{mM}-\mathrm{L}$-glutamine (Sigma), 
$50 \mu \mathrm{M}$ 2-mercaptoethanol (Sigma), $30 \mu \mathrm{g} / \mathrm{ml}$ gentamycin (Sigma), and $100 \mathrm{ml} / \mathrm{l}$ normal human serum, in the presence of PHA (Sigma) or ConA (Sigma), both at 1 and $5 \mu \mathrm{g} / \mathrm{ml}$. Normal human serum was a pool of sera from five healthy department members; these sera had all been tested individually in pilot experiments, and were free of detectable mitogenic activity. They were heat inactivated by incubation at $56^{\circ}$ for exactly $30 \mathrm{~min}$ followed by $15 \mathrm{~min}$ centrifugation at $1500 \mathrm{rev} . / \mathrm{min}$ before storage. On each culture plate control wells were included with pre- and post-intervention PBMC of the same subject in the same medium without mitogens.

After incubation for $90 \mathrm{~h}$ at $37^{\circ}, 5 \% \mathrm{CO}_{2}$ and $95-98 \%$ relative humidity, cells were washed twice in cold serum-free saline, and resuspended in the original volume $(0.5 \mathrm{ml})$ of RPMI-1640, with glutamine, gentamycin and mercaptoethanol, but without human serum. In these suspensions metabolic cell activity was assessed by the MTT method (Mosmann, 1983 ) with adaptations taken from Denizot \& Lang (1986). Thus, of each $0.5 \mathrm{ml}$ suspension three $0.12 \mathrm{ml}$ portions were applied to a ninety-six-well flat-bottom plate, mixed with $10 \mu \mathrm{l}$ (10 mg/l) solution of 3,4,5-dimethylthiazol-2-yl)-2,5-diphenyl tetrazolium bromide (MTT; Sigma) stock solution. After $4 \mathrm{~h}$ incubation $120 \mu \mathrm{l}$ isopropanol was added to each well to stop MTT conversion and to dissolve the produced formazan, which was quantified spectrophotometrically at $570 \mathrm{~nm}$.

Mitogenic stimulation was expressed as the difference in $A_{570}$ between the mean of duplicate cells cultured with mitogens and the control cell cultures.

\section{Data analysis}

Baseline results are expressed as means and standard deviations (SD) or median and 25$75 \%$ range. Mean baseline values and mean individual changes during intervention were compared between the study groups by using the unpaired $t$ test in the case of normal distribution and the Mann-Whitney test. Similarly, mean individual changes over the intervention period for the control and vitamin $\mathrm{E}$ groups were tested with the paired $t$ test or with the Wilcoxon-signed rank test for paired comparisons within groups.

Multiple linear regression was used to adjust differences in change in proliferative response values between the placebo group and the vitamin E group for initial values (Chambless \& Roeback, 1993). Presented correlations are expressed as Spearman correlation coefficients. All analyses were performed using the statistical package SAS (Statistical Analysis Systems, 1989).

\section{RESULTS}

Table 1 presents the mean baseline characteristics of the placebo and vitamin Esupplemented groups. Pre-stratification on sex, smoking behaviour and 5-year age groups was successful, as shown by equal distribution of these variables in both study groups. Subjects participated for, on average, 3 months in the study ( $84 \mathrm{~d}$; Table 1$)$ with a variation from 61 to $94 \mathrm{~d}$. Differences in supplementation period, mainly due to measuring during the holiday period (May-June), were not associated with immune response nor with particular population characteristics. The number of subjects not reporting any intercurrent illness or medicine use during the trial was slightly higher in the vitamin E group ( $n$ 21) than in the control group ( $n$ 16).

Baseline plasma antioxidant vitamin concentrations and estimated dietary antioxidant vitamin intake did not differ significantly between intervention and control groups (Table 2). In the vitamin $\mathrm{E}$ group plasma $\alpha$-tocopherol increased during intervention by $51 \%$ (mean 
Table 1. Characteristics of elderly subjects participating in a 3-month intervention trial with vitamin $E$

(Mean values with their standard deviations or frequency $(\%)$ )

\begin{tabular}{|c|c|c|c|c|c|c|}
\hline & \multicolumn{3}{|c|}{ Control group ( $n$ 36) } & \multicolumn{3}{|c|}{ Vitamin E group ( $n$ 38) } \\
\hline & Mean & & SD & Mean & & SD \\
\hline Age (years) & 74.5 & & $5 \cdot 2$ & $74 \cdot 2$ & & $5 \cdot 2$ \\
\hline Male $(\%)$ & & 67 & & & 61 & \\
\hline Smokers $(\%)$ & & 25 & & & 24 & \\
\hline Intervention period (d) & $83 \cdot 7$ & & 6.8 & $84 \cdot 2$ & & 4.5 \\
\hline Pills taken $(\%)$ & $93 \cdot 9$ & & 9.0 & 93.0 & & 6.9 \\
\hline Health score* & $8 \cdot 2$ & & $1 \cdot 3$ & 8.2 & & $1 \cdot 3$ \\
\hline Height (m) & 1.70 & & 0.09 & 1.70 & & 0.09 \\
\hline $\mathrm{BMI}\left(\mathrm{kg} / \mathrm{m}^{2}\right)$ & $25 \cdot 1$ & & $3 \cdot 2$ & 25.9 & & $3 \cdot 2$ \\
\hline No reported illness or medicine use $f(\%)$ & & 44 & & & 55 & \\
\hline Reported illness (d) $\dagger_{+}^{+}$ & $5 \cdot 5$ & & 8.7 & 3.5 & & 3.4 \\
\hline
\end{tabular}

* Rating on a scale from 1 (worst) to 10 (best).

$\dagger$ Based on self-reported occurrence of illness and use of medicine, recorded in a health diary during the 3-month intervention period.

$\ddagger$ Only for the group who reported illness or medicine use in their diary: placebo group, $n$ 20, and vitamin $\mathrm{E}$ group, $n 17$.

$16.7(\mathrm{SD} 12.7) \mu \mathrm{mol} / 1, P=0.0001)$. In the vitamin $\mathrm{E}$ group the increase in $\alpha$-tocopherol was correlated with an observed decrease of plasma $\beta+\gamma$-tocopherol (Spearman correlation $r-0.5, P=0.006)$. At baseline only one subject had a plasma $\alpha$-tocopherol level below the cut-off value of $11.6 \mu \mathrm{mol} / 1$ for risk of vitamin $E$ deficiency in human subjects and none below the $2.2 \mu \mathrm{mol} \alpha$-tocopherol/mmol cholesterol value. Taking the optimal levels to prevent chronic disease as postulated by Gey (1994), $32 \%$ fell below the $30 \mu \mathrm{mol}$ plasma $\alpha$ tocopherol $/ 1$ limit and $18 \%$ below the $5.2 \mu \mathrm{mol} \alpha$-tocopherol $/ \mathrm{mmol}$ cholesterol value. In this study similar values for the reported immune variables were observed between subjects above and below the optimal $\alpha$-tocopherol levels mentioned earlier.

\section{Humoral immune response; immunoglobulin $G$ and $A$ antibody concentrations}

At baseline, median concentrations of IgA and IgG raised against Penicillium and IgG4 raised against three different food antigens were comparable in vitamin $E$ and placebo groups (Table 3). In the vitamin E-supplemented group no significant changes in antibody concentrations were observed after 3 months. In the control group a small but significant increase was seen for the IgG concentration against Penicillium (median change $\mathrm{A}_{492} \mathbf{0} \cdot \mathbf{1}$; $P=0.01$ ) and a small decrease in IgG4 against milk protein (median change $\mathrm{A}_{492}-0.01$; $P=0.04$ ). During the intervention period, similar changes were observed in the intervention and placebo groups. Baseline and 3-month antibody concentrations were highly correlated ( $r \quad 0.9$ for all antibody concentrations in both groups). Baseline concentrations of IgG4 against the three different food antigens showed strong mutual correlations $(0.6<r<0.7 ; P=0.0001)$.

Baseline antibody concentrations for the total study population were not significantly correlated with age, BMI, antioxidant plasma concentrations or antioxidant vitamin intake. Higher baseline values of IgG4 against food antigens were seen for females than for males (IgG4 against milk protein: median $\mathrm{A}_{492} 2.6(25-75 \%$ interval 0.3-4.3) for females and 0.5 (interval $0.03-2.2$ ) for males, $P=0.03$ ). These sex differences persisted when comparing 


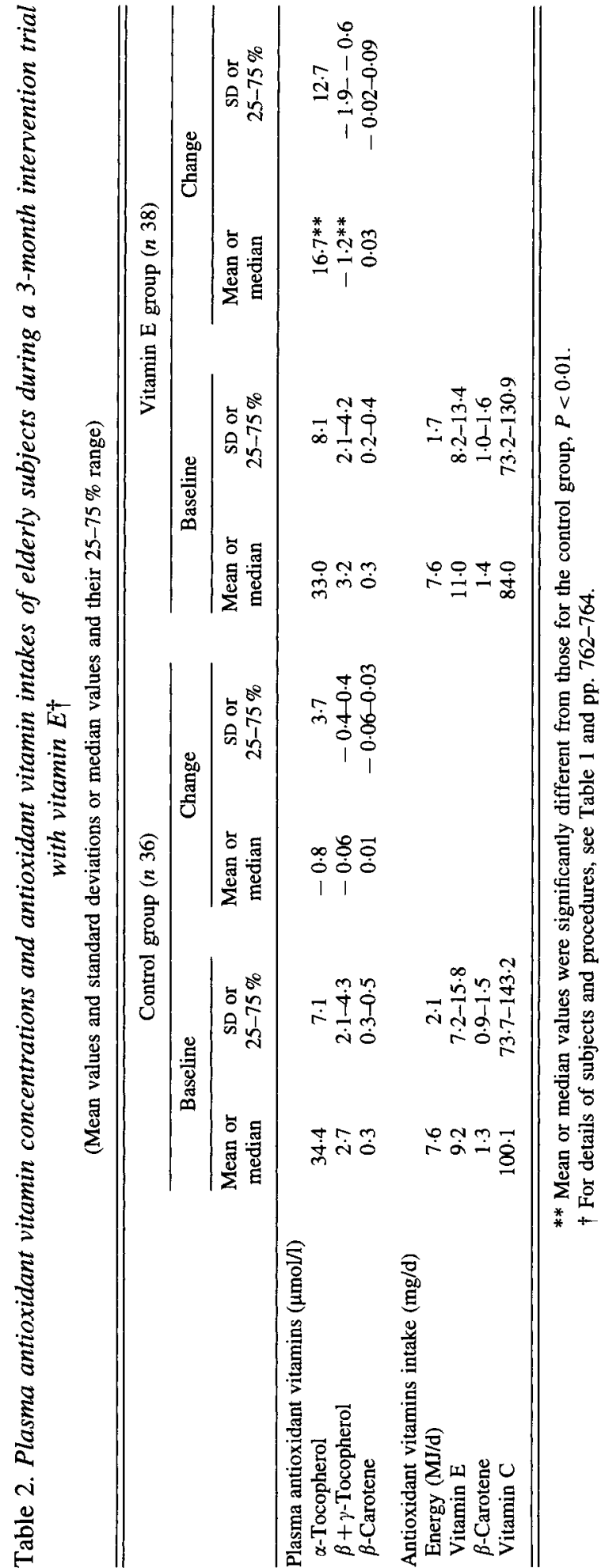


Table 3. Concentrations of antibodies against common antigens (absorbance at $492 \mathrm{~nm}$ ) in elderly subjects and changes to these concentrations during a 3-month intervention trial with vitamin $E^{\dagger}$

(Median values and $25-75 \%$ range)

\begin{tabular}{|c|c|c|c|c|c|c|c|c|}
\hline & \multicolumn{4}{|c|}{ Control group ( $n$ 36) } & \multicolumn{4}{|c|}{ Vitamin E group ( $n 38$ ) } \\
\hline & \multicolumn{2}{|c|}{ Baseline } & \multicolumn{2}{|c|}{ Change } & \multicolumn{2}{|c|}{ Baseline } & \multicolumn{2}{|c|}{ Change } \\
\hline & Median & $25-75 \%$ & Median & $25-75 \%$ & Median & $25-75 \%$ & Median & $25-75 \%$ \\
\hline IgA, Penicillium & $1 \cdot 2$ & $0.5-2 \cdot 0$ & 0.01 & $-0.1-0.1$ & 1.0 & $0.6-2 \cdot 2$ & -0.01 & $-0.1-0.1$ \\
\hline IgG, Penicillium & $2 \cdot 8$ & $1.5-3.6$ & $0 \cdot 1^{*}$ & $-0.0-0.3$ & $3 \cdot 0$ & $2.4-4.0$ & -0.04 & $-0.2-0.2$ \\
\hline IgG4, egg protein & $3 \cdot 3$ & $2 \cdot 1-5 \cdot 5$ & -0.1 & $-0.3-0.3$ & 3.9 & $0.6-5.8$ & 0.02 & $-0.1-0.2$ \\
\hline IgG4, milk protein & $1 \cdot 1$ & $0 \cdot 1-3 \cdot 1$ & $-0.01 *$ & $-0.2-0.0$ & 1.0 & $0 \cdot 0-4 \cdot 2$ & $0 \cdot 0$ & $-0 \cdot 1-0.1$ \\
\hline IgG4, wheat protein & 0.2 & $0.0-2.6$ & $0 \cdot 0$ & $-0.1-0.0$ & 0.5 & $0 \cdot 1-2 \cdot 4$ & $0 \cdot 0$ & $-0 \cdot 1-0.1$ \\
\hline
\end{tabular}

Ig, immunoglobulin.

* Changes were significantly different from zero, $P<0.05$.

$\dagger$ For details of subjects and procedures, see Table 1 and pp. 762-765.

non-smoking males ( $n$ 30) and females ( $n$ 26). No sex differences for $\operatorname{IgG}$ and $\operatorname{IgA}$ raised against Penicillium were observed. The difference between smokers and non-smokers was evaluated among men because only one female smoker was present. Only for IgG against Penicillium was a significantly $(P=0.0004)$ lower baseline value for smokers (median 2.1 (interval 1.2-2.7)) seen as compared with non-smokers (median 3.5 (interval 2.7-4.3)).

\section{Cellular immune response; mitogen stimulation of peripheral blood mononuclear cells with phytohaemagglutinin and concanavalin $A$}

Data for cellular immune responses were available for a subpopulation of fifty-two subjects from the total study population. Baseline characteristics of this subpopulation were similar to those given for the total study population in Tables 1 and 2 .

At baseline lymphocyte proliferation to both mitogens ConA and PHA $(5 \mu \mathrm{g} / \mathrm{ml})$ was significantly lower $(P=0.04)$ in the control group (Table 4). Furthermore, changes in PHA proliferative responses during intervention were negatively correlated with initial values ( $r-0.5, P<0.02$ for both study groups). To obtain an unbiased estimate, differences in change in proliferative response between the vitamin $E$ group and the placebo group were adjusted for initial proliferative response values. As shown in Table 4 the adjusted treatment effect indicates that vitamin $E$ had no effect on mitogenic stimulation of PBMC with PHA and ConA. To explore a potential effect of baseline $\alpha$-tocopherol plasma values on immune response we analysed separately the subjects in the lower two tertiles of baseline $\alpha$-tocopherol values. This left thirty-two subjects ( $n 16$ for both control and vitamin $E$ groups) for analysis, with mean $\alpha$-tocopherol baseline values of 28.9 (SD 4.9) $\mu \mathrm{mol} / \mathrm{l}$ and 27.5 (SD $5.6 \mu \mathrm{mol} / \mathrm{l}$ ) and increases during intervention of 0.4 (SD 3.4) and 11.2 (SD 9.6) $\mu \mathrm{mol} / 1$ in the control and vitamin $\mathrm{E}$ groups respectively. The results on mitogenic response for this sub-sample did not differ from those obtained from the total sample.

$\mathrm{A}_{570}$ values for unstimulated PBMC were similar for control and vitamin $\mathrm{E}$ group showing means of 0.10 (SD 0.05) and 0.11 (SD 0.08) respectively. Correlations between baseline and post-intervention measurements for PHA-stimulated PBMC were $r 0.5$ $(P=0.006)$ and $r 0.8(P=0.0001)$ for control and vitamin $\mathrm{E}$ groups respectively. For ConA, correlations of $r 0.6(P=0.0008)$ and $r 0.7(P=0.0001)$ were observed (see Fig. 1$)$. 


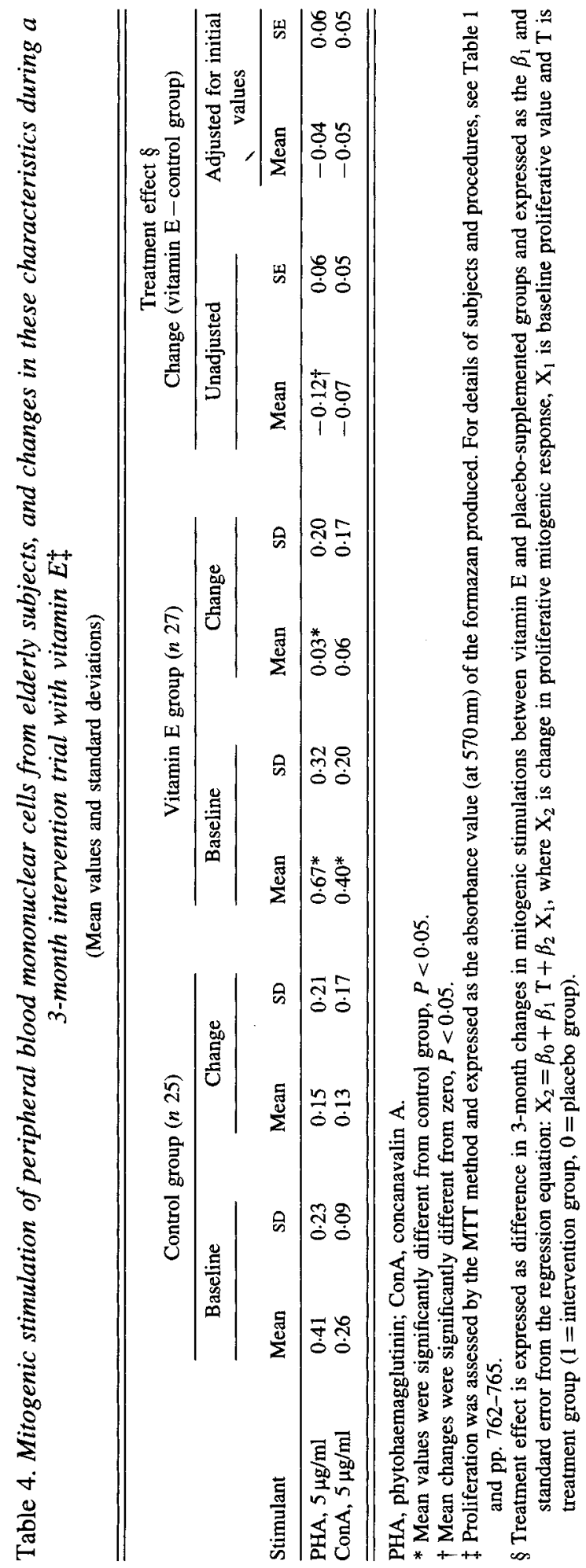



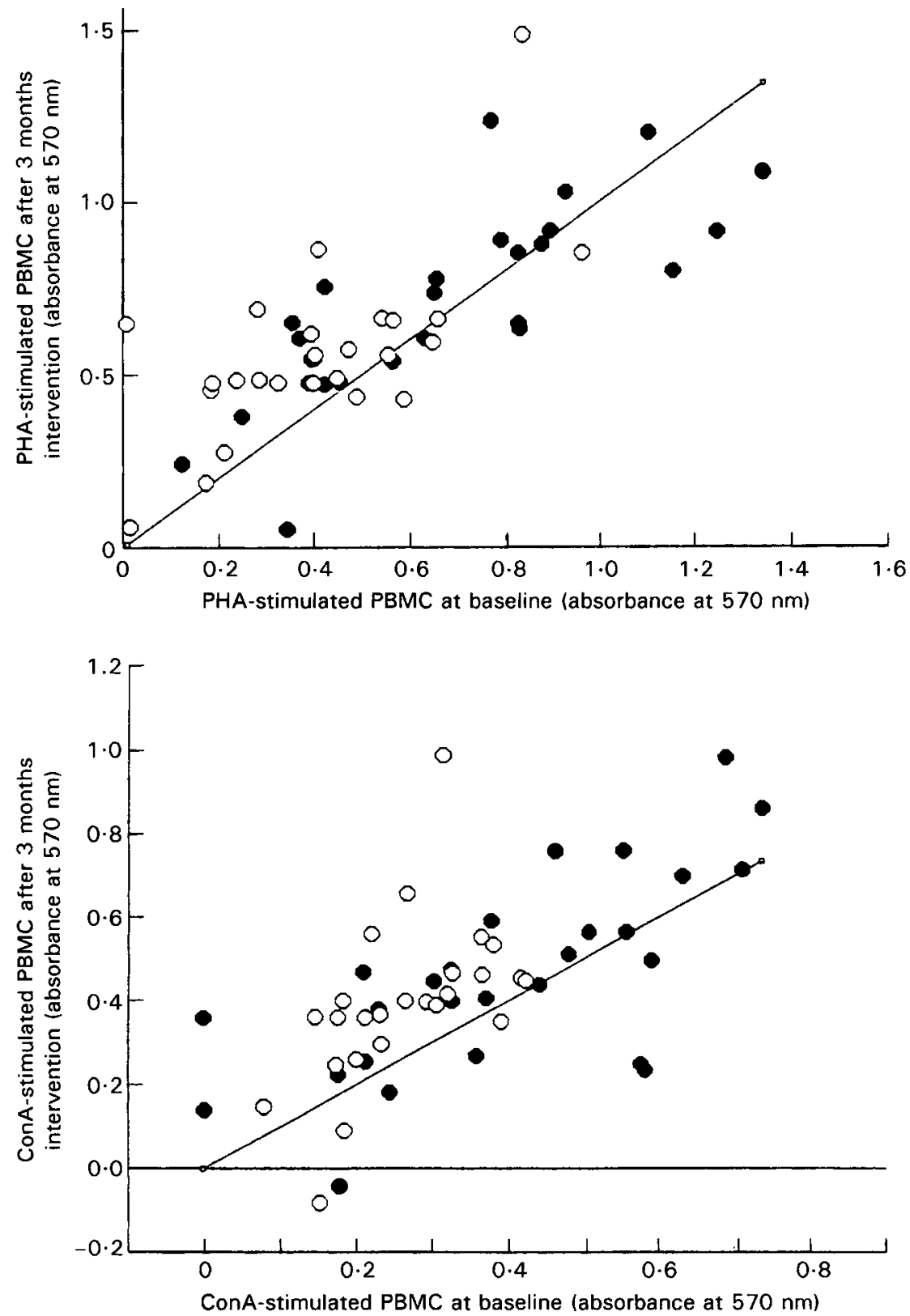

Fig. 1. Stimulation of peripheral blood mononuclear cells (PBMC) from elderly subjects with the mitogens phytohaemagglutinin (PHA) and concanavalin A (ConA) measured at baseline $v$. measurements made after 3 months intervention with vitamin $\mathrm{E}(\bigcirc)$ or a placebo $(\bigcirc)$. For Spearman correlation coefficients, see pp. 768-771. Proliferation was assessed by the MTT method and expressed as the absorbance at $570 \mathrm{~nm}$ of the formazan produced. The line shown is the plot $\mathrm{X}=\mathrm{Y}$. 
Baseline values for proliferative response were not significantly correlated with age, BMI, antioxidant plasma concentrations or antioxidant vitamin intake. No differences were found between men and women or between smokers and non-smokers in baseline values.

Similar results were observed for proliferative responses with $1 \mu \mathrm{g}$ mitogen/l (results not shown).

\section{DISCUSSION}

In this 3-month intervention trial among apparently healthy elderly subjects no effect was observed of $100 \mathrm{mg} \alpha$-tocopheryl acetate on the in vitro proliferative response of PBMC to the mitogens PHA and ConA. Also antibody concentrations against various common antigens were not affected by vitamin $E$ supplementation.

Ageing itself, and diseases that frequently accompany ageing, are thought to be factors explaining the decline in immune responsiveness with ageing. Goodwin et al. (1982) found no difference in cellular immune responsiveness between healthy and chronically ill elderly subjects. This suggests that the major determinant of decreased cellular immune function is age per se and not age-associated diseases. Therefore improving immune responsiveness in healthy elderly people may be of great public health importance. The present study population was selected as 'healthy' using a questionnaire on disease and medicine use, and therefore cannot be considered representative of the elderly population in general. Underlying subclinical diseases may well have been present but are not expected to have influenced the comparison between the two study groups because of randomization. Comparison within study groups of those not reporting illness during intervention with those reporting illness, did not reveal any differences in studied immune variables.

A great variability inherent to the mitogen proliferation assays has been mentioned by Meydani et al. (1990). To avoid inter-assay variation, isolated PBMC were stored in liquid $\mathrm{N}_{2}$ and mitogen proliferative responses of pre- and post-intervention PBMC for each individual were measured simultaneously in the same run. The storage period of PBMC was not significantly associated with the proliferative responses.

Intra-individual variation in the mitogenic response measured at different time points may lead to regression towards the mean and could have affected our results, as we observed unexpected differences in initial values in mitogenic response between vitamin $E$ and control groups. Baseline PHA and ConA responses in the control group were significantly lower than in the vitamin $\mathrm{E}$ group, while post-intervention responses were similar in both groups and at the level of the vitamin E group at baseline. Therefore we adjusted the intervention effect (changes in proliferative response between vitamin $E$ and control group) for initial values by means of multiple linear regression (Chambless \& Roeback, 1993).

Vitamin $\mathrm{E}$ is thought to exert its effect on $\mathrm{T}$-cell response and interleukin-2 production. Therefore an effect on humoral immune response would be primarily expected for T-celldependent antibody responses. Ziemlanski et al. (1986) and Meydani et al. (1990) did not show an effect of vitamin $\mathrm{E}$ on total antibody (IgG, $\operatorname{IgM}, \operatorname{IgA}, \operatorname{IgD})$ concentrations. Total IgG serum concentrations are, however, a rather crude estimate of humoral immune responsiveness. Therefore, we decided to measure specific antibodies to various common antigens like Penicillium, milk, wheat and egg proteins to which most individuals are probably constantly exposed, and against which specific IgG and $\operatorname{IgA}$ antibodies are produced. 
Taking into account the reported steady state of plasma $\alpha$-tocopherol after 4-5 d following chronic administration of vitamin $\mathrm{E}$ (Dimitrov et al. 1991), the lifetime of activated B-cells ( 4 weeks) and the half-life of antibodies in circulation (3 weeks), the intervention period of 3 months seems adequate for detecting important changes in antibody production.

Improvement of indices of the cellular and humoral immune response by vitamin $\mathrm{E}$ has been extensively demonstrated in animal experiments. Reviews on the subject (e.g. Meydani et al. 1995) indicate important implications for the human situation, although this is supported by only a few studies in human populations. The first intervention study among elderly people on the effect of vitamin $E$ on a large number of cellular and humoral immune indices was reported by Meydani et al. (1990). They showed increases in the proliferative response of PBMC to ConA but not to PHA after daily supplementation with $800 \mathrm{mg} \alpha$-tocopheryl acetate for $30 \mathrm{~d}$. The rise in plasma $\alpha$-tocopherol in their study was $3-$ fold while in our study with $100 \mathrm{mg} \alpha$-tocopheryl acetate the relative increase was $51 \%$. The smaller increase in $\alpha$-tocopherol in blood and probably also in PBMC (not measured in the present study but in the study by Meydani et al. (1990) also 3-fold) might not have been enough to improve the in vitro mitogen proliferation of PBMC. This could mean that very high vitamin $E$ doses are needed to improve mitogen-stimulated proliferation of PBMC. This is supported by another intervention in elderly subjects reported by Meydani et al. (1994), with administration of different vitamin $E$ doses $(60,200$ and $800 \mathrm{mg}$ vitamin $E$ for $120 \mathrm{~d}$ ), and in which a significant increase in mitogenic response to ConA was observed for the $800 \mathrm{mg}$ dose only. An increased response to delayed-type hypersensitivity tests was, however, seen in all supplemented groups.

Alternatively, our results may be due partly to the relatively high baseline plasma $\alpha$ tocopherol values in the present study as compared with, for example, the trial of Meydani et al. (1990). Chandra (1992) found, in a 1 year multivitamin supplementation (including $44 \mathrm{mg}$ vitamin $E$ ) trial among healthy elderly subjects, that improvement in immunological responses (increase in natural killer cells, lymphocyte proliferation to PHA, a higher antibody response to influenza vaccine, and a lower frequency of infection-related illness) was greater among subjects who at baseline had low plasma nutrient values. Therefore we tested the intervention effect for the subjects in the lower two tertiles of the baseline plasma values revealing similar mean plasma $\alpha$-tocopherol values to those in the study of Meydani et al. (1990) but still found no effect on mitogenic response between the control and the vitamin $\mathrm{E}$ group. Therefore, it does not seem likely that relatively high baseline vitamin $\mathrm{E}$ levels are to be held responsible for not finding an effect of vitamin E supplementation on cellular immune response in the present study.

Our study in the elderly did not show improvements in specific immune indices after supplementation with $100 \mathrm{mg} \alpha$-tocopheryl acetate for 3 months. This does not mean that relatively low doses of vitamin $E$ might not affect other immune variables in elderly subjects (see Meydani et al. 1994). Studies on different doses of vitamin E and different immune variables will improve the available information on the overall possible positive effects and working mechanism and should provide necessary background information on the optimal doses. Better insight into improvement of the immune responsiveness in elderly people by vitamin $\mathrm{E}$ might warrant studies relating vitamin $\mathrm{E}$ to morbidity and mortality, especially those factors contributing to infections.

We wish to thank the elderly volunteers for their enthusiastic participation in this study. The part of the study on cell-mediated immune response was financially supported by Nutricia Research. 


\section{REFERENCES}

Bendich, A., Gabriel, E. \& Machlin, L. J. (1986). Dietary vitamin E requirement for optimum immune responses in the rat. Journal of Nutrition 116, 675-681.

Boyum, A. (1968). Isolation of mononuclear cells and granulocytes from human blood. Scandinavian Journal of Clinical and Laboratory Investigation 21 Suppl., 77-89.

Burns, E. A., Lum, L. G., Seigneuret, M. C., Giddings, B. R. \& Goodwin, J. S. (1990). Decreased specific antibody synthesis in old adults: decreased potency of antigen-specific B cells with ageing. Mechanisms of Ageing and Development 53, 229-241.

Chambless, L. E. \& Roeback, J. R. (1993). Methods for assessing difference between groups in change when initial measurement is subject to intra-individual variation. Statistics in Medicine 12, 1213-1237.

Chandra, R. K. (1992). Effect of vitamin and trace-element supplementation on immune responses and infection in elderly subjects. Lancet 340, 1124-1127.

Chavance, M., Herbeth, B., Fournier, C., Janot, C. \& Vernhes, G. (1989). Vitamin status, immunity and infections in an elderly population. European Journal of Clinical Nutrition 43, 827-835.

Corwin, L. M., Gordon, R. K. \& Shloss, J. (1981). Studies of the mode of action of vitamin E in stimulating Tcell mitogenesis. Scandinavian Journal of Immunology 14, 565-571.

Corwin, L. M. \& Shloss, J. (1980a). Role of antioxidants on the stimulation of the mitogenic response. Journal of Nutrition 110, 2497-2505.

Corwin, L. M. \& Shloss, J. (1980b). Influence of vitamin $\mathrm{E}$ on the mitogenic response of murine lymphoid cells. Journal of Nutrition 110, 916-923.

Denizot, F. \& Lang, R. (1986). Rapid colorimetric assay for cell growth and survival. Modification to the tetrazolium dye procedure giving improved sensitivity and reliability. Joumal of Immunological Methods 89, 271-277.

Dimitrov, N. V., Meyer, C., Gilliland, D., Ruppenthal, M., Chenoweth, W. \& Malone, W. (1991). Plasma tocopherol concentrations in response to supplemental vitamin E. American Journal of Clinical Nutrition 53, 723-739.

Doekes, G., Kaal, M. J. H. \& Van Ieperen-van Dijk, A. G. (1993). Allergens of Pityrosporum ovale and Candida albicans. II. Physicochemical characterization. Allergy 48, 401-408.

Gardner, I. D. (1980). The effect of aging on susceptibility to infection. Reviews of Infectious Diseases 2, 801810.

Gey, F. K. (1994). Optimum plasma levels of antioxidant micronutrients. Ten years of antioxidant hypothesis on atherosclerosis. Bibliotheca Nutrito et Dieta 51, 84-99.

Goetzel, E. J. (1980). Vitamin E modulates the lipoxygenation of arachidonic acid in leukocytes. Nature $\mathbf{2 8 8}$, 183-185.

Goodwin, J. S. (1995). Decreased immunity and increased morbidity in the elderly. Nutrition Reviews 53, S41S46.

Goodwin, J. S. \& Garry, P. J. (1983). Relationship between megadose vitamin supplementation and immunological function in a healthy elderly population. Clinical Experimental Immunology 51, 647-653.

Goodwin, J. S., Searles, R. P. \& Tung, S. K. (1982). Immunological responses of a healthy elderly population. Clinical Experimental Immunology 48, 403-410.

Hayek, M. G., Meydani, S. N., Meydani, M. \& Blumberg, J. B. (1994). Age differences in eicosanoid production of mouse splenocytes: effects on mitogen-induced T-cell proliferation. Journal of Gerontology 49, B197B207.

Hess, D., Keller, H. E., Oberlin, B., Bonfanti, R. \& Schuep, W (1991). Simultaneous determination of retinol, tocopherols, carotenes and lycopene in plasma by means of high performance liquid chromatography on reversed phase. International Journal of Vitamin and Nutrition Research 61, 232-238.

Houba, R., VanRun, P., Heederik, D. \& Doekes, G. (1996). Wheat antigen exposure assessment for epidemiologic studies in bakeries using personal sampling and inhibition ELISA. Clinical and Experimental Allergy 26, 154-163.

Kishimoto, S., Tomino, S., Mitsuya, H., Fujiwara, H. \& Tsuda, H. (1980). Age-related decline in the in vitro and in vivo syntheses of anti-tetanus toxoid antibody in humans. Journal of Immunology 125, 2347-2352.

Larsen, H. J. \& Tollersrud, S. (1981). Effect of dietary vitamin E and selenium on the phytohaemagglutinin response of pig lymphocytes. Research in Veterinary Science 31, 301-305.

Likoff, R. O., Guptill, D. R., Lawrence, L. M., McKay, C. C., Mathias, M. M., Nockels, C. F. \& Tengerdy, R. P. (1981). Vitamin E and aspirin depress prostaglandins in protection of chickens against Escherichia coli infection. American Journal of Clinical Nutrition 34, 245-251.

Makinodan, T. (1995). Patterns of age-related immunologic changes. Nutrition Reviews 53, S27-S34.

Meydani, S. N., Barklund, M. P., Liu, S., Meydani, M., Miller, R. A., Cannon, J. G., Morrow, F. D., Rocklin, R. \& Blumberg, J. B. (1990). Vitamin E supplementation enhances cell-mediated immunity in healthy elderly subjects. American Journal of Clinical Nutrition 52, 557-563.

Meydani, S. N., Leka, L. \& Loszewski, R. (1994). Long term vitamin E supplementation enhances immune response in healthy elderly. FASEB Journal 8, A272, Abstr. 1574. 
Meydani, S. N., Meydani, M., Verdon, C. P., Shapiro, A. A., Blumberg, J. B. \& Hayes, K. C. (1986). Vitamin E supplementation suppresses prostaglandin E2 synthesis and enhances the immune response of aged mice. Mechanisms of Ageing and Development 34, 191-201.

Meydani, S. N., Wu, D. Y., Santos, N. S. \& Hayek, M. G. (1995). Antioxidants and immune response in aged persons, overview of present evidence. American Journal of Clinical Nutrition 62, S1462-S1476.

Mosmann, T. (1983). Rapid colorimetric assay for cellular growth and survival: application to proliferation and cytotoxicity assays. Journal of Immunological Methods 65, 55-63.

Murasko, D. M., Weiner, P. \& Kaye, D. (1988). Association of lack of mitogen-induced lymphocyte proliferation with increased mortality in the elderly. Aging: Immunology and Infectious Disease 1, 1-6.

Ocké, M., Bueno de Mesquita, B., Pols, M., Smit, H., Van Staveren, W. \& Kromhout, D. (1997). The Dutch EPIC food frequency questionnaire. II. Relative validity and reproducibility for nutrients. International Journal of Epidemiology 26, F49-F58.

Payette, H., Rola-Pleszczynski, M. \& Ghadirian, P. (1990). Nutrition factors in relation to cellular and regulatory immune variables in a free-living elderly population. American Journal of Clinical Nutrition 52, 927-932.

Reddy, P. G., Morrill, J. L., Minocha, H. C. \& Stevenson, J. S. (1987). Vitamin E is immunostimulatory in calves. Journal of Dairy Science 70, 993-999.

Roberts-Thomson, I. C., Whittingham, S., Youngchaiyud, U. \& Mackay, I. R. (1974). Ageing, immune response, and mortality. Lancet ii, $368-370$.

Statistical Analysis Systems (1989). SAS User's Guide, version 6, 4th ed., vol. 1/2. Cary, NC : SAS Institute Inc.

Tengerdy, R. P., Heinzerling, R. H., Brown, G. L. \& Mathias, M. M. (1973). Enhancement of the humoral immune response by vitamin E. International Archives of Allergy and Applied Immunology 44, 221-232.

Tengerdy, R. P., Mathias, M. M. \& Nockels, C. F. (1984). Effect of vitamin E on immunity and disease resistance. In Vitamins, Nutrition and Cancer, pp. 123-133 [K. N. Prasad, editor]. Basel: Karger.

Van den Homberg, C. E. J. (1995). Physical activity and health characteristics; a survey among Dutch elderly women and men. PhD Thesis, Agricultural University Wageningen, pp. 5-17. Den Haag: CIP-data Koninklijke Bibliotheek.

Wayne, S. J., Rhyne, R. L., Garry, P. J. \& Goodwin, J. S. (1990). Cell-mediated immunity as predictor of morbidity and mortality in subjects over 60. Journal of Gerontology 45, M45-M48.

Weksler, M. E. \& Hutteroth, T. H. (1974). Impaired lymphocyte function in aged humans. Journal of Clinical Investigation 53, 99-104.

Ziemlanski, S., Wartanowicz, M., Klos, A., Raczka, A. \& Klos, M. (1986). The effects of ascorbic acid and $\alpha-$ tocopherol supplementation on serum proteins and immunoglobulin concentrations in the elderly. Nutrition International 2, 245-249.

Zock, J. P., Doekes, G., Heederik, D., VanZuylen, M. \& Wielaard, P. (1996). Airborne dust antigen exposure and specific IgG response in the potato processing industry. Clinical and Experimental Allergy 26, 542-548. 